\title{
Control System Development for an Advanced-Technology Medium-Duty Hybrid Electric Truck
}

\author{
Chan-Chiao Lin, Huei Peng and Jessy W. Grizzle \\ University of Michigan \\ Jason Liu and Matt Busdiecker \\ Eaton Corporation
}

Copyright $@ 2003$ SAE International

\begin{abstract}
The power management control system development and vehicle test results for a medium-duty hybrid electric truck are reported in this paper. The design procedure adopted is a model-based approach, and is based on the dynamic programming technique. A vehicle model is first developed, and the optimal control actions to maximize fuel economy are then obtained by the dynamic programming method. A near-optimal control strategy is subsequently extracted and implemented using a rapid-prototyping control development system, which provides a convenient environment to adjust the control algorithms and accommodate various $1 / O$ configurations. Dynamometer-testing results confirm that the proposed algorithm helps the prototype hybrid truck to achieve a $45 \%$ fuel economy improvement on the benchmark (non-hybrid) vehicle. It also compares favorably to a conventional rule-based control method, which only achieves a $31 \%$ fuel economy improvement on the same hybrid vehicle.
\end{abstract}

\section{INTRODUCTION}

Hybrid powertrain is among the most visible transportation technologies developed over the last decade. Starting from the ground-breaking PNGV effort in the early 1990's, the introduction of Prius and Insight hybrid vehicles in the late 1990's, to the planned 2005 lineup of close to 10 commercially available vehicles in the US, hybrid vehicles have moved quickly from concept to reality. This quick acceptance is mainly due to the potential of hybrid technologies in reducing fuel consumption and emissions, especially for vehicles driving in urban areas with frequent starts and stops.

In this paper, the design of a power management control system is described for a hybrid electric vehicle (HEV). The hybrid electric truck that employs this control system features a "Direct Hybrid" powertrain system [1], which integrates an advanced diesel engine, an electric traction motor, Lithium-Ion batteries, an automatic clutch, and an automated manual transmission system. The motor is directly linked between the output of the master clutch and the input to the transmission. This architecture provides the regenerative braking during deceleration and allows efficient motor assist and recharge operations by the engine.

The control of hybrid powertrains is more complicated than the control of ICE-only powertrain. First, one needs to determine the optimal operating mode among five possible modes (motor only, engine only, power assist, recharge, and regenerative). Furthermore, when the power assist mode or the recharge mode is selected, the engine power, motor power and transmission gear ratio need to be selected to achieve optimal fuel economy, emissions reduction, charge balance, and drivability. With the increased powertrain complexity and the need to achieve multiple objectives, a two-level control architecture was adopted. A supervisory powertrain controller (SPC) sits at the top to manage the operation of the hybrid powertrain system. The supervisory powertrain controller is designed to include the following functions: power management strategy, transmissions shifting control, smooth operation logic, I/O communication, and system monitor and diagnosis. At every sampling time, the supervisory powertrain controller sends commands (set points or desired states) to each sub-system control module and receives sensor signals and diagnostic status from each sub-system. The low-level control systems manipulate the local-level inputs to follow the SPC commands as long as other local constraints are not violated.

To ensure that the SPC achieves a guaranteed level of performance and robustness, a model-based design process was adopted. First, models and look-up tables for all sub-systems were developed or documented. A vehicle simulation model was then developed for vehicle performance analysis and control algorithm development. The SPC control was developed based on the dynamic programming technique, which aims to 
International Truck \& Bus Meeting \& Exhibition, Fort Worth, TX, November 2003.

maximize fuel economy without sacrificing drivability. A near-optimal control strategy was extracted and implemented in a PC-based rapid-prototyping system to provide a fast and easy way to adjust the control algorithms and accommodate various $\mathrm{l} / \mathrm{O}$ configurations. More importantly, the entire development process of the control system provides a seamless environment of control algorithm design, implementation, and testing for flexible hybrid powertrains.

The paper is arranged as follows. The configuration of the prototype hybrid electric vehicle system is introduced first, followed by the description of the control system architecture implemented in the vehicle. Next, a modelbased design approach based on the dynamic programming technique is proposed to develop the control strategy in the supervisory powertrain controller. The prototype hybrid vehicle with the developed control system is evaluated through the chassis dynamometer test to demonstrate the fuel economy improvement, followed by the summary and conclusion.

\section{PROTOTYPE HYBRID TRUCK INTEGRATION}

The goal of the prototype hybrid truck program is to improve the fuel economy by $50 \%$ and to reduce emissions by $90 \%$ over the benchmark, non-hybrid vehicle. In order to achieve this aggressive performance requirement, the function of the hybrid system needs to be optimized to provide these large improvements in fuel economy and emissions, while still meeting the performance requirements of the vehicle.

\section{VEHICLE SYSTEM CONFIGURATION}

The hybrid electric powertrain, shown in Figure 1 is a parallel hybrid configuration that has the capability to provide five different operational modes: motor-only, engine-only, power-assist, recharging, and regenerative braking. The down-sized diesel engine is connected to the automatic clutch which is electronically controlled to smoothly engage and disengage during the vehicle launch and stop scenarios. The electric motor is directly mounted on the output of the automatic clutch. In other words, the engine and electric motor both transfer power to the output of the automatic clutch, and no additional torque coupling device is required in this configuration. The blended torque of the engine and motor drives the Eaton Fuller AutoShift transmission, which is a shift-bywire automated manual transmission (AMT) system. This allows gear shifting operation without driver involvement, similar to an automatic transmission, while possessing the high efficiency of a manual transmission. Figure 2 shows the Hybrid Drive Unit assembly, which incorporates the automatic clutch, electric motor, and automated manual transmission into a single driveline component.

It should be noted that the chassis and body of the baseline truck were modified only minimally to enable the hybridization. There were no changes to major chassis systems such as brakes, wheels and tires. The basic specifications of the vehicle are given in Table 1.

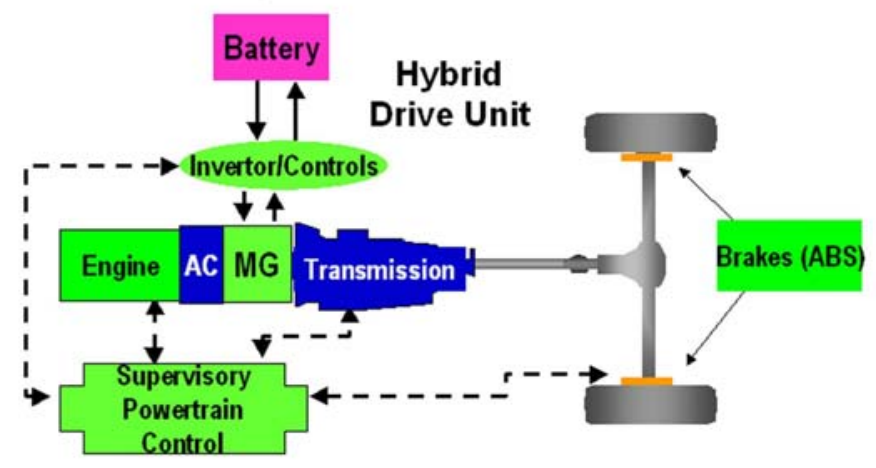

Figure 1: Eaton hybrid electric powertrain

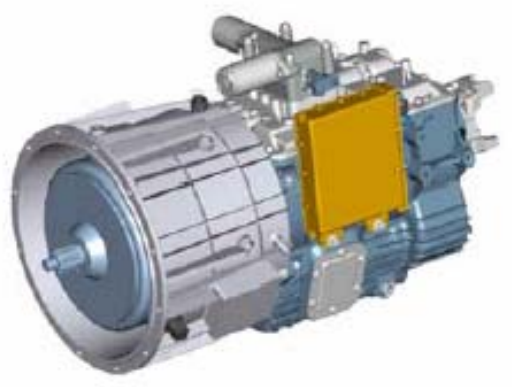

Figure 2: Hybrid Drive Unit assembly

Table 1: Basic vehicle specifications

\begin{tabular}{|l|l|}
\hline Engine & I4, 4.3L, 170HP \\
\hline Transmission & $\begin{array}{l}\text { 6 speed, Automated } \\
\text { Manual }\end{array}$ \\
\hline Electric Motor & $\begin{array}{l}\text { Peak Power: } 44 \mathrm{~kW} \\
\text { Peak Torque: } 420 \mathrm{Nm}\end{array}$ \\
\hline Battery & $\begin{array}{l}\text { Li-Ion type } \\
\text { Nominal Voltage: } 340 \mathrm{~V} \\
\text { Energy Storage: } 2.5 \mathrm{kWh}\end{array}$ \\
\hline Wheels & 19.5 inch, steel \\
\hline GVWR & $16000 \mathrm{lbs}$ \\
\hline Cargo Area & 700 cubic feet \\
\hline Rear Axle Ratio & 3.31 \\
\hline
\end{tabular}

\section{CONTROL SYSTEM ARCHITECTURE}

The hybrid vehicle is an integrated system that consists of many sub-systems including engine, transmission, motor, battery, clutch, brakes, etc. Each sub-system is 
International Truck \& Bus Meeting \& Exhibition, Fort Worth, TX, November 2003.

also a complex system that has its own functionality and desired performance. In this case, almost every subsystem is equipped with sensors, actuators, and a control system to regulate its behavior. Moreover, all sub-systems need to be coordinated in an optimal manner to achieve different objectives, e.g. fuel economy, emissions reduction, charge balance, and drivability. With this increasing complexity of powertrain system and the need of achieving multiple objectives, an integrated vehicle-level controller is required to accomplish the task [2].

\section{TWO-LEVEL HIERARCHICAL CONTROL ARCHITECTURE}

A two-level hierarchical control architecture is used in controlling the prototype hybrid powertrain as shown in Figure 3 . The supervisory powertrain controller (SPC) is an electronic control unit that controls the operation of the hybrid system through multiple inputs and outputs, monitors the system status, and manages communication with other on-board systems. In the twolevel control architecture, the SPC is regarded as a highlevel vehicle control system that coordinates the overall powertrain to satisfy certain performance target such as fuel economy and emissions reduction. Based on driver's demand (e.g. accelerator and brake pedal signals) and current state of the sub-systems (e.g. engine speed, motor speed, SOC, etc.), the high-level powertrain controller must determine the desired output to be generated by the sub-systems (e.g. engine torque, motor torque, requested gear, etc.). These desired output signals are sent to the corresponding subsystems and become the commands for the low-level control system of each sub-system. These low-level control systems include engine electronic control unit (ECU), motor controller, transmission controller, and battery controller, which are normally provided by subsystem supplier/OEM. A Controller Area Network (CAN) bus provides communications between the supervisory control system and each low-level control system.

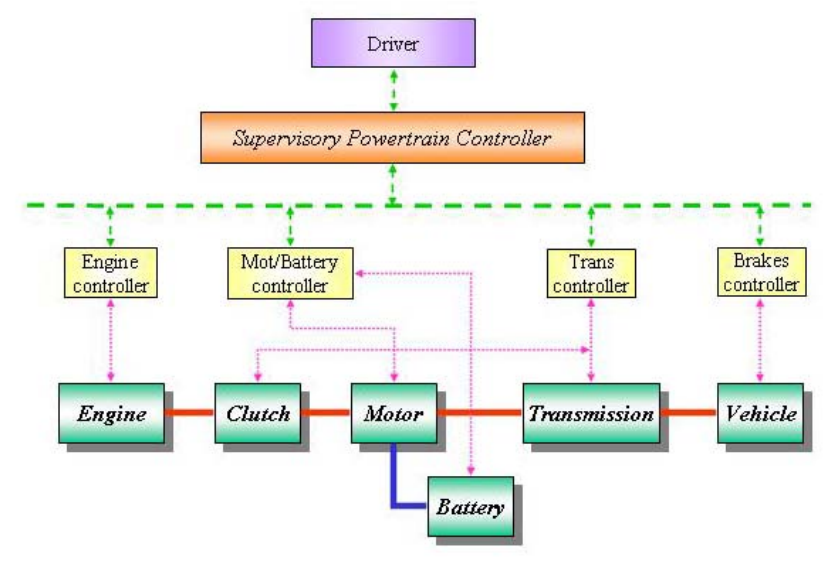

Figure 3: Hierarchical control architecture of a hybrid electric vehicle
For most cases, the task of the low-level controller can be treated as a classical regulating/tracking control problem. The low-level control systems can also be designed for different goals, such as improved drivability, while ensuring the set-points commanded by the high-level controller are achieved reliably. The twolevel control architecture implies that the supervisory controller only controls the hybrid vehicle by using highlevel control signals such as power, torque, and speed while the low-level variables such as fuel injection, current, and voltage are kept within the low-level controllers. This makes it possible to simplify and expedite the control design. It should be noted that much attention has been paid to the design of the subsystem controllers due to the dominance of conventional vehicles and continuing research on electric vehicles. The related technologies are relatively mature. However, a systematic design approach for the highlevel control system in hybrid vehicles is still not generally available, and needs to be developed.

\section{SUPERVISORY POWERTRAIN CONTROLLER}

In this study, we concentrate on the development of supervisory control strategy for the hybrid vehicle. The command from the supervisory controller is assumed to be perfectly executed within the next time step. In order to achieve the desired performance in fuel economy, emissions reduction, drivability, and safety, the supervisory powertrain control system needs to include the following key functions regarding the optimal operation of the energy conversion and storage devices: power management strategy, regenerative braking control, transmission shift logic and shifting control, vehicle launch control, and system fault detection. These functions are partitioned into modules and carefully designed to achieve desired performance. The major functions are described as follows.

\section{$\underline{\text { Regenerative braking control }}$}

Regenerative braking is one of the key advantages of hybrid vehicles. The kinetic energy normally dissipated during braking can be recaptured by applying negative torque to the electric motor. Since the regenerative braking and traditional friction braking co-exist in this vehicle architecture, the coordination between these two braking systems to achieve driver's braking demand is a main function of the regenerative braking control. Because of the fact that the hydraulic friction braking in the prototype vehicle is not electrically controlled, a parallel braking system that can simultaneously apply friction braking and regenerative braking is used. The driver brake input corresponds directly to the friction braking torque since the friction brake line is directly connected to the brake pedal. The amount of regenerative braking torque that can be added to the friction braking torque is calculated by considering the electric motor torque characteristics, vehicle speed, and driver feel. 
International Truck \& Bus Meeting \& Exhibition, Fort Worth, TX, November 2003.

Transmission shift logic and shifting control

The gear position of the transmission has a significant influence on fuel economy and emissions because it influences the operating point of the engine. This simple fact is sometimes overlooked in the design of control strategies for hybrid vehicles. In the supervisory control system, the requested gear position is a control signal sent to the transmission control system. This requested gear command is determined by a gear shifting logic based on the vehicle status information such as input shaft speed of the transmission, current gear position, and driver pedal command. Most of the existing literatures use a heuristic approach [3] or static optimization [4] to design the shift logic for hybrid vehicles. However, in order to improve fuel economy, emissions, drivability, and shifting quality simultaneously, the shift logic requires an integrated design approach by considering the overall hybrid powertrain (engine, electric motor, energy storage, and transmission) together.

Coordinating the hybrid powertrain to accomplish the gear-shift process of the automated manual transmission is another important task of the SPC. The related control functions in the SPC include the clutch engage/disengage control, and torque/speed control of the engine and the motor. The gear shifting in the AMT is controlled by the transmission controller. The control of the entire gear-shifting process is designed to ensure the shift duration and shift shock are minimized

\section{Power management strategy}

The power management strategy in the SPC is crucial for balancing between efficiency and performance of hybrid vehicles. The term "power management" refers to the design of the higher-level control algorithm that determines the proper power (torque) level to be generated, and its split between the motor and the engine while satisfying the power (torque) demand from the driver and maintaining adequate energy in the energy storage device. It should be noted that the power management could be either a torque-based or a power-based strategy depending on the application. Since the engine ECU and motor controller both accept the torque command, the torque-based strategy is used in this study.

Many existing power management strategies employ heuristic control techniques such as control rules/fuzzy logic for the control algorithm development. The idea of this approach is based on the concept of "load-leveling", which attempts to operate the irreversible energy conversion device such as an internal combustion engine (ICE) or fuel cell (FC) in an efficient region and uses the reversible energy storage device as a loadleveling device, to compensate the rest of the power demand. Due to the unknown nature of future power demand, a charge sustaining strategy is needed to maintain the state of charge (SOC) level in the load- leveling device. The thermostat SOC strategy, in which the SOC is cycled between low and high limit, was often used due to its simplicity and robustness [5]. Another popular strategy is to adopt a rule-based structure by defining a set of thresholds to implement in the control logic [6]. The thresholds could then be identified through a optimization process or tuned by a set of simulations over a given driving cycle. There has been much other research on implementation of load-leveling and chargesustaining strategy by using fuzzy logic technique [7 and 8]. The fuzzy logic concept is essentially a rule-based system that relies on intuition and heuristics to identify the controller. Another popular approach is based on the static optimization method that decides the proper split between the two energy sources by minimizing the total equivalent consumption cost [3]. Generally, the electric power is translated into an equivalent amount of (steady-state) fuel rate in order to calculate the overall fuel cost. Another effective approach is to use dynamic optimization technique that considers the dynamic nature of the system when performing the optimization [9].

The output of the power management strategy is the motor torque command and engine torque command, which are designed for the purposes of fuel economy and drivability. These torque commands are normally sent directly to the sub-system controllers, e.g. motor ECU and engine ECU. However, the torque commands will be overridden under certain conditions. One situation is when there exists a sub-system fault. For example, if a battery fault exists, the torque commands from the power management will be bypassed and the SPC will request the engine to satisfy the driver demand as much as possible. Another example is that during a gear shift, the torque/speed commands generated by the shifting control function will override the command from the power management strategy.

\section{SUPERVISORY CONTROL STRATEGY DESIGN}

The main objective of the supervisory control strategy design is to develop a near-optimal and practical power management strategy that determines the proper torque split and gear selection for the prototype hybrid truck to minimize the fuel consumption at all times; meanwhile, it also satisfies the following constraints.

- Meet the power demand from the driver.

- Maintain state of charge of the energy storage device.

- Achieve certain drivability requirements.

Moreover, the design procedure is required to be systematic, accommodating multiple objectives, costeffective, and re-useable. In this study, we use a modelbased design approach based on the simulation and dynamic optimization to extract implementable, nearoptimal control rules, which are then implemented in the vehicle by using a rapid prototyping tool. The control 
International Truck \& Bus Meeting \& Exhibition, Fort Worth, TX, November 2003.

strategy could be tested and tuned in the simulation environment, hardware-in-the-loop (HIL), and field test in a fast and cost-effective way.

\section{SIMULATION MODEL DEVELOPMENT}

The first step of the model-based design process is to develop a simulation model for the hybrid electric vehicle. The vehicle model is constructed to directly resemble the layout of the physical system. In order to have a high degree of flexibility, the model is implemented in the Matlab/Simulink/Stateflow software environment. Links between main modules represent the physical variables that actually define the interaction between the components, such as shaft torque and angular velocity, or electrical current and voltage. A feed-forward simulation scheme is employed so as to enable studies of control strategies under realistic transient conditions, where everything starts with the driver action and the "pedal position" signal being sent to the supervisory powertrain controller. The HEV controller contains the power management logic and sends control signals to the component modules based on the feedback about current operating conditions. Finally, a "driver" module was built to allow the feed-forward simulation in order to follow a prescribed vehicle speed schedule. The driver controller fulfills that role and provides the driver throttle demand signal and braking request, based on the specified speed setting and the current vehicle speed

The driving cycle used in the simulation as well as the final chassis dynamometer test is shown Figure 4. This test cycle was provided as being typical of the normal operation of this vehicle, and can be described as a modified version of the 1975 Federal Test Procedure (FTP) test cycle. Only the first 1372 seconds of the FTP is used and an engine shutdown at every other vehicle stop is added to represent this particular pick-up and delivery vehicle application.

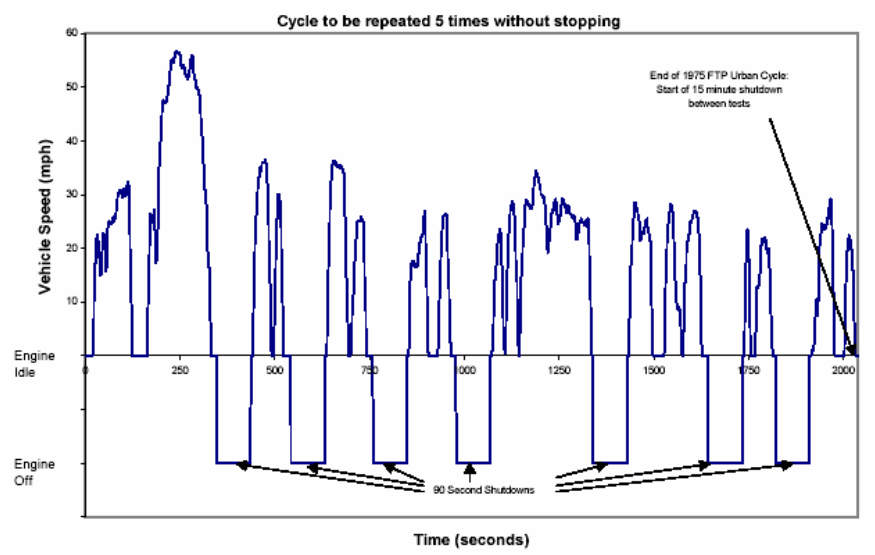

Figure 4: Customized delivery truck test cycle
It is known that the main control challenge for HEV is to determine the proper operation mode, the power/torque split ratio between the two power sources and the gearshifting schedule. Control strategies based on engineering intuition or trial-and-error commonly fail to achieve satisfactory improvement due to the complex nature of HEV dynamics and the trade-off among multiple objectives (fuel economy, emissions and drivability). In this section, a design procedure based on the Dynamic Programming technique for the design of a near-optimal control strategy is described.

\section{Dynamic Optimization Problem Formulation}

The control of HEV is formulated as an optimal control problem in the Dynamic Programming approach [9]. The goal is to find a sequence of control actions, including the engine torque, motor torque, and gear selection, of the hybrid powertrain to minimize a cost function, which is the sum of fuel consumption for a defined driving cycle:

$$
\min J=\min _{\left\{T_{e, k}, T_{m, k}, g_{k}\right\}, k=0,1, . . N-1} \sum_{k=0}^{N-1} W_{f u e l, k}
$$

where $k$ is the index of the time step, $N$ is the total number of steps of the driving cycle, $W_{\text {fuel }, k}$ is the engine fuel flow rate, and the time step is chosen to be long enough that all these "commands" can be executed by the servo-loop controllers and that no frequent changes from the main loop controller will happen. For this work, we chose to have the time step at one second.

\section{System Equations}

Once a driving cycle is given (e.g., Figure 4), the wheel torque $T_{w h, r e q}$ required to follow the speed profile can be determined for each time step by inversely solving the vehicle dynamics. The corresponding wheel speed $\omega_{w h, r e q}$ can be computed by feeding the required wheel torque to the vehicle model in order to include the wheel dynamics and the slip effect. At every time step, the torque balance equation in the wheel needs to be satisfied as follows

$$
T_{w h, k}\left(T_{e, k}+T_{m, k}, g_{k}, \omega_{w h, r e q, k}\right)+T_{b r a k e, k}=T_{w h, r e q, k}
$$

where $T_{w h, k}$ is the wheel torque propagated from the sum of the engine torque, $T_{e, k}$, and motor torque, $T_{m, k}$, through the driveline, $g_{k}$ is the transmission gear number, and $T_{\text {brake, } k}$ is the friction braking torque. A simplified hybrid powertrain dynamic model can be represented as a discrete-time dynamic system.

$$
S O C_{k+1}=S O C_{k}+f\left(S O C_{k}, T_{m, k}, \omega_{m, k}\right)
$$


International Truck \& Bus Meeting \& Exhibition, Fort Worth, TX, November 2003.

$$
g_{k+1}= \begin{cases}6, & g_{k}+\text { shift }_{k}>6 \\ 1, & g_{k}+\text { shift }_{k}<1 \\ g_{k}+\text { shift }_{k}, & \text { otherwise }\end{cases}
$$

It can be seen that there are only two state variables: the battery state of charge, $S O C_{k}$, and the transmission gear number, $g_{k}$. The control inputs to this dynamic system are the engine torque, $T_{e, k}$ and gear shifting command, shift $t_{k}$, which is constrained to take on the values of $-1,0$, and 1 , representing downshift, hold and up-shift, respectively. It should be noted that the motor torque becomes a dependent variable instead of a control variable due to the driveline torque balance constraint.

\section{Inequality Constraints}

During the optimization procedure, it is necessary to impose inequality constraints to ensure safe and smooth operation of the engine, motor, and battery. In general, these practical considerations can be written in mathematical form as

$$
\begin{aligned}
& \omega_{e_{-} \min } \leq \omega_{e, k} \leq \omega_{e_{-} \max } \\
& T_{e_{-} \min }\left(\omega_{e, k}\right) \leq T_{e, k} \leq T_{e_{-} \max }\left(\omega_{e, k}\right) \\
& T_{m_{-} \min }\left(\omega_{m, k}, S O C_{k}\right) \leq T_{m, k} \leq T_{m_{-} \max }\left(\omega_{m, k}, S O C_{k}\right) \\
& S O C_{\min } \leq S O C_{k} \leq S O C_{\max }
\end{aligned}
$$

where $\omega_{e}$ is the engine speed, and the battery SOC limits, $S O C_{\min }$ and $S O C_{\max }$ are 0.4 and 0.7, respectively, which are recommended by the battery manufacturer. Besides, performing gearshifts on grades is critical to the automated manual transmission. In order to reduce the possibility of missing synchronization, the restriction of the gear shifting selection is also taken into account in the optimization as follows

$$
\begin{cases}\omega_{w h, r e q, k} \cdot R_{f} \cdot R_{g}\left(g_{k+1}\right)>\omega_{i n_{-} \min }, & \text { if } \text { shift }_{k}=1 \\ \omega_{w h, r e q, k} \cdot R_{f} \cdot R_{g}\left(g_{k+1}\right)<\omega_{\text {in_max }}, & \text { if } \text { shift }_{k}=-1\end{cases}
$$

where $\omega_{i n_{-} \min }$ and $\omega_{i n_{-} \max }$ are minimum and maximum allowable input shaft speed, respectively. $R_{f}$ is the gear ratio of the final drive, and $R_{g}$ is the gear ratio of the transmission.

\section{Augmented Cost Function}

The basic power management problem stated above does not contain any constraint to limit the use of electric energy. The optimization algorithm has a tendency to deplete the battery to attain minimal fuel consumption. Therefore, a terminal penalty on SOC is introduced to maintain the battery energy.

$$
G_{N}\left(S O C_{N}\right)=\alpha\left(S O C_{N}-S O C_{d}\right)^{2}
$$

where $S O C_{d}$ is the desired SOC at the end time of the cycle and $\alpha$ is the weighting factor. The purpose is to ensure that the SOC is moved back to its desired value at the end of the driving cycle.

In addition, the minimization of the fuel consumption without considering dynamic constraints of the gear would result in frequent gear shifting, which is unfavorable to the transmission and also undesirable to the driver. Hence, an extra term that penalizes the use of gear changes is defined

$$
L_{k}=\beta \cdot\left|g_{k+1}-g_{k}\right|
$$

By adding Eqs. (7) and (8) into the original cost function, the augmented cost function becomes

$$
\min J=\min _{\left\{T_{e, k}, s h i f_{k}\right\}, k=0,1, \ldots N-1}\left\{\sum_{k=0}^{N-1}\left[W_{f u e l, k}+L_{k}\right]+G_{N}\right\} .
$$

\section{Dynamic Programming Results}

Dynamic programming (DP) is a powerful tool to solve general dynamic optimization problems. Its main advantage is that it can handle constraints and nonlinearities while obtaining an optimal solution. The DP technique is based on Bellman's Principle of Optimality, which states that the optimal policy can be obtained if we first solve a one stage sub-problem involving only the last stage and then gradually extend to sub-problems involving the last two stages, last three stages, ...etc. until the entire problem is solved. In this manner, the overall dynamic optimization problem can be decomposed into a sequence of simpler minimization problems as follows [10]

Step $N-1$ :

$$
J_{N-1}^{*}\left(S O C_{N-1}, g_{N-1}\right)=\min _{T_{e, N-1}, \text { shift } t_{N-1}}\left[W_{\text {fuel }, N-1}+L_{N-1}+G_{N}\left(S O C_{N}\right)\right]
$$

Step $k$, for $0 \leq k<N-1$

$$
J^{*}{ }_{k}\left(S O C_{k}, g_{k}\right)=\min _{T_{e, k}, s h i f_{k}}\left[W_{\text {fuel }, k}+L_{k}+J^{*}{ }_{k+1}\left(S O C_{k+1}, g_{k+1}\right)\right]
$$

where $J_{k}^{*}\left(S O C_{k}, g_{k}\right)$ represents the optimal cost-to-go function or optimal value function at state $S O C_{k}$ and $g_{k}$ starting from time stage $k$. The above recursive equation is solved backwards to find the optimal control policy. The minimizations are performed subject to the inequality constraints shown in Eqs. (5) and (6).

The DP procedure described above produces an optimal, time-varying state-feedback control law, i.e. 
International Truck \& Bus Meeting \& Exhibition, Fort Worth, TX, November 2003.

$u_{k}^{*}\left(S O C_{k}, g_{k}\right)$. This optimal control policy can then be used to drive the hybrid vehicle along an optimal trajectory such that the cost function in (9) is minimized. Simulation results under the optimal DP policy are shown in Figure 5. The engine power and motor power trajectories represent the optimal operation between two power movers to achieve the best fuel economy. The initial condition of SOC and gear position in the simulation are 0.6 and first gear, respectively. Since the final desired SOC in Eq. (7) was selected to be 0.6, the simulation shows the SOC trajectory returns to 0.6 at the end of the cycle.
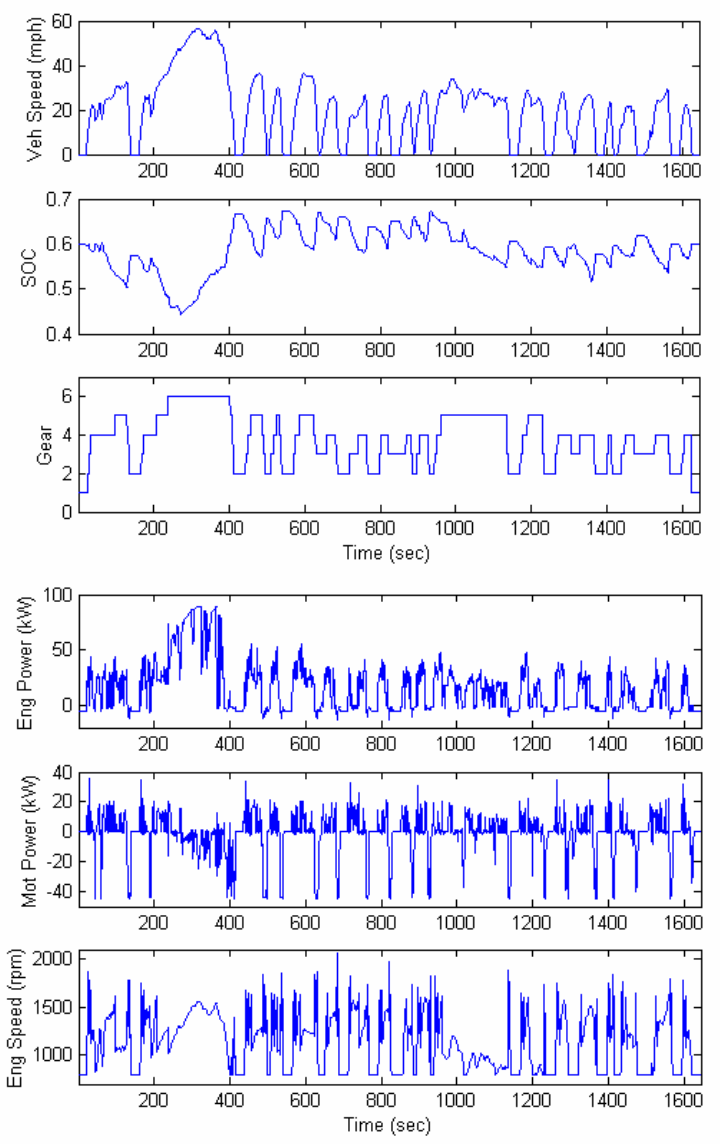

Figure 5: DP simulation results

\section{RULE-BASED CONTROL STRATEGY IMPLEMENTATION}

Although the Dynamic Programming approach provides an optimal solution, the resulting control policy is not implementable under real driving conditions because it requires the knowledge of future speed and load profile. The result is, on the other hand, a benchmark which other control strategies can be compared to and learn from. Therefore, the second part of the control design procedure involves knowledge extraction from DP results to obtain implementable rule-based control algorithms (Figure 6). Overall, the behaviors to learn include the transmission gearshift logic and the power- management strategy. The gearshift logic was found to be crucial for the fuel economy of hybrid electric vehicles. From the DP results, the optimal gear operational points and upshift/downshift points are plotted on the standard transmission shift-map to identify an optimal shifting schedule as shown in Figure 7 . The identified shifting schedule defines the optimal upshift and downshift thresholds to the gear selection control unit, which is implemented in Simulink/Stateflow (Figure 7).

There are four possible operating modes of splitting the power demand between the engine and the motor when the driver requests a positive power demand: motor-only mode, engine-only mode, power-assist mode (both the engine and motor), and recharge mode (the engine offers additional power to charge the accumulator). Rules for switching between different modes are established by examining the optimization results obtained from dynamic programming. The optimal operating points displaying different operating modes are superimposed on the engine BSFC map given in the engine power vs. speed diagram (see Figure 8). The control rules of switching operation modes and the torque split ratio can then be extracted and implemented in the Simulink/Stateflow environment.

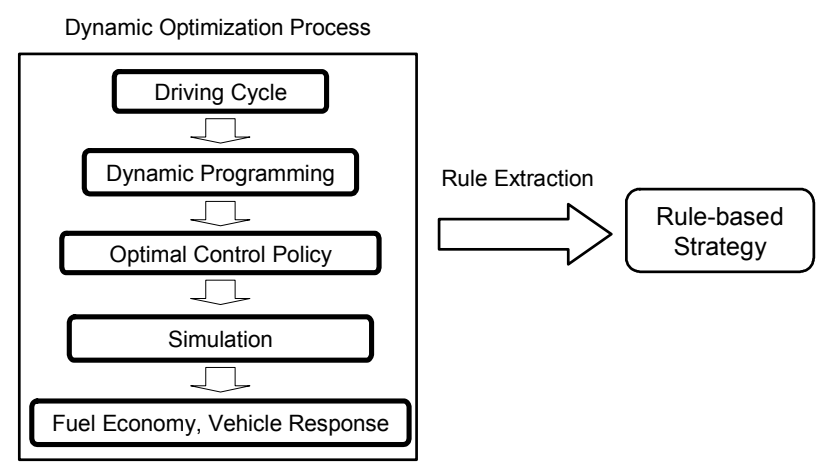

Figure 6: DP-based design process

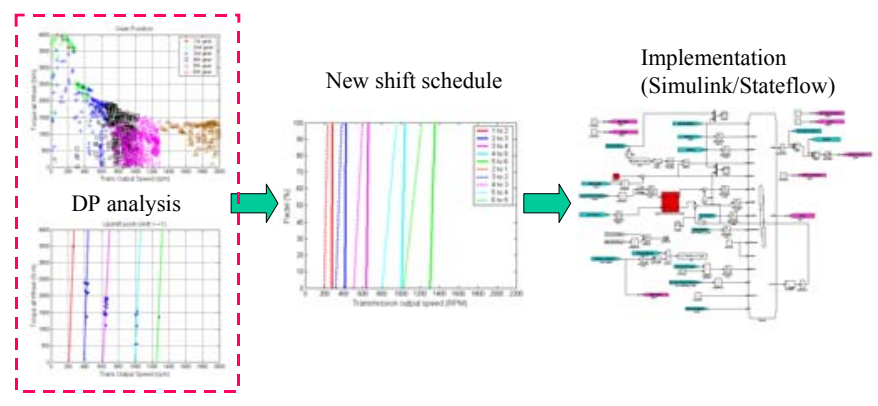

Figure 7: Optimal gearshift logic 


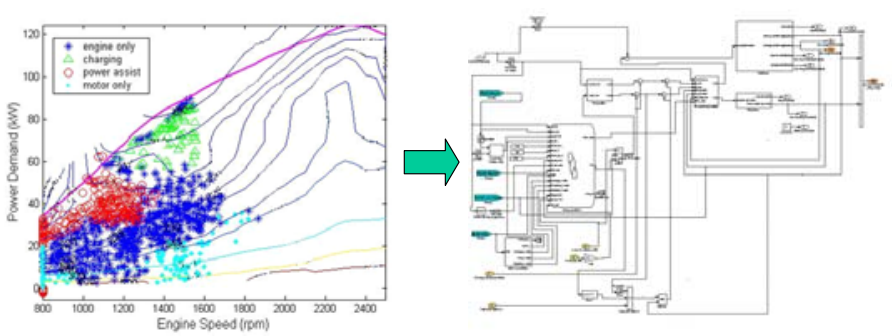

Figure 8: Optimal power split strategy

\section{CONTROL SYSTEM TESTING AND CALIBRATION}

Once the supervisory powertrain controller is developed, the control system can be tested and redesigned in phases as shown in Figure 9. The SPC developed in the previous section can be first evaluated by using the simulation model. This simulation phase allows the algorithms and parameters in the SPC to be examined and tuned before the hardware prototype is available. In order to reduce the development time and cost, Eaton used a PC-based rapid control prototyping tool, to implement the SPC in the prototype vehicle. The Simulink/Stateflow-based SPC model on the host computer can be built and downloaded to the supervisory control computer on the prototype vehicle via an Ethernet connection. The prototype vehicle computer consisted of a $400 \mathrm{MHz} \mathrm{CPU}$, an A/D board, an Ethernet card, a D/A board, two CAN boards, a timer/counter board, and a power supply board. An LCD display is used as the target display to the driver. Realtime data can be captured using a host computer and plotted for later analysis. This rapid prototyping system enables the engineers not only to test and operate the real components in the Hardware-in-the-loop phase, but also to test the vehicle on the road in a real driving phase. From the real-time measurement, the engineers could quickly analyze the performance of the SPC, modify the controller model, and build and download the modified code to prototype vehicle computer in a fast and cost-effective manner.

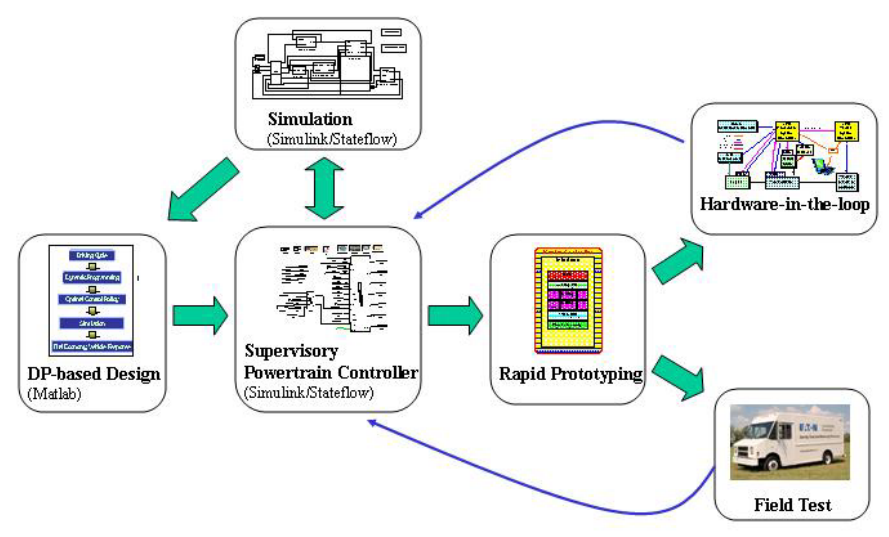

Figure 9: Model-based control design iteration

\section{CHASSIS DYNAMOMETER TESTING RESULTS}

The SPC algorithm is implemented on the prototype vehicle (for details, please see the companion paper "Class 4 Hybrid Truck for Pick Up and Delivery Applications" [1]). Emissions and fuel economy measurements were made on a full-scale chassis dynamometer, and the results from 5 repetition runs are averaged and reported in Table 2. In Table 2, the "baseline control strategy" refers to a classic loadleveling type rule-based algorithm implemented on the same hybrid truck. The "DP-based control strategy" refers to the near-optimal rule-based strategy trained from DP data. The highlights of the results are that while it is very important to install good hardware (battery, motor, etc.) and to choose a smaller and more efficient engine, it is also very important to carefully design the power management algorithm. A simple rulebased algorithm which takes advantage of only engine operation efficiency results in a $31 \%$ fuel economy improvement (over existing technology, ICE-engine only truck). However, proper software change (with no real add-on cost) brings about another $14 \%$ of improvement.

Table 2: Dynamometer testing results over the modified FTP cycle

\begin{tabular}{|l|l|l|}
\hline $\begin{array}{l}\text { Comparison of } \\
\text { Eaton hybrid truck } \\
\text { to the baseline } \\
\text { truck }\end{array}$ & $\begin{array}{l}\text { Baseline control } \\
\text { strategy }\end{array}$ & $\begin{array}{l}\text { DP-based control } \\
\text { strategy }\end{array}$ \\
\hline $\begin{array}{l}\text { Fuel Economy } \\
\text { (MPG) }\end{array}$ & $31 \%$ increase & $45 \%$ increase \\
\hline NOx (g/mile) & $50 \%$ reduction & $54 \%$ reduction \\
\hline
\end{tabular}

\section{CONCLUSION}

The design of the power management strategy for HEV by extracting rules from the Dynamic Programming results has the advantage of being model-based, systematic, and near-optimal. By solving, and analyzing the DP results, an improved rule-based control strategy 
International Truck \& Bus Meeting \& Exhibition, Fort Worth, TX, November 2003.

was developed and implemented on a prototype medium-duty truck produced by the Eaton Corporation. Dynamometer test results show that the proposed design procedure resulted in a high-performance control algorithm. The fuel economy of the hybrid electric truck was found to be $45 \%$ higher over the ICE-only benchmark truck, compared to a $31 \%$ fuel economy improvement obtained with conventional controls on the same hybrid vehicle.

\section{ACKNOWLEDGMENTS}

This power management application utilized principles that were developed during earlier research done at the Automotive Research Center of the University of Michigan, and was supported by the U.S. Army TARDEC under the contract DAAE07-98-C-R-L008.

\section{REFERENCES}

1. Nellums, R., Steffen, J., and Naito, S., "Class 4 Hybrid Truck for Pick Up and Delivery Applications," SAE International Truck \& Bus Meeting \& Exhibition, SAE Paper 2003-01-3368, November, 2003.

2. Phillips, A., Jankovic, M., and Bailey, K., "Vehicle System Controller Design for a Hybrid Electric Vehicle," Proceedings of the 2000 IEEE International Conference on Control Applications, Alaska, September, 2000.

3. Paganelli, G., Ercole, G., Brahma, A., Guezennec, Y. and Rizzoni, G., "General Supervisory Control Policy for the Energy Optimization of Charge-Sustaining Hybrid Electric Vehicles." JSAE Review, Vol. 22, 2001, pp.511-518.

4. Lee, H.-D., Sul, S.K., Cho, H.S., and Lee, J.M., "Advanced Gear Shifting and Clutching Strategy for Parallel Hybrid Vehicle with Automated Manual Transmission," IEEE Industry Applications Conference, 1998.

5. Rahman, Z., Butler, K., and Ehsani, M., "A Comparison Study Between Two Parallel hybrid Control Concepts," SAE Paper, No. 2000-01-0994, 2000.

6. Wipke, K., Cuddy, M., and Burch, S., "ADVISOR 2.1: a user-friendly advanced powertrain simulation using a combined backward/forward approach," IEEE Transaction on Vehicular Technology, Vol. 48, No. 6, 1999, pp. 1751-1761.

7. Baumann, B. M., Washington, G. N., Glenn, B. C., and Rizzoni, G., "Mechatronic Design and Control of Hybrid Electric Vehicles," IEEE/ASME Transactions on Mechatronics, v5 n 1 2000. p 58-72, 2000.

8. Schouten, N., Salman, M., Kheir, N., "Fuzzy Logic Control for Parallel Hybrid Vehicles," IEEE Transactions on Control Systems Technology, Vol. 10, No. 3, May 2002, pp.460-468.

9. Lin, C.-C., Kang, J., Grizzle, J. W. and Peng, H., "Energy Management Strategy for a Parallel Hybrid Electric Truck," Proceedings of the 2001 American
Control Conference, Arlington, VA, June, 2001, pp.2878-2883.

10.Bertsekas, D. P., Dynamic Programming and Optimal Control, Athena Scientific, 1995

\section{DEFINITIONS, ACRONYMS, ABBREVIATIONS}

\section{Abbreviations}

AMT: Automated Manual Transmission

CAN: Controller Area Network

DP: Dynamic Programming

ECU: Electronic Control Unit

HEV: Hybrid Electric Vehicle

ICE: Internal Combustion Engine

SPC: Supervisory Powertrain Controller

SOC: Battery State of Charge 
International Truck \& Bus Meeting \& Exhibition, Fort Worth, TX, November 2003. 